Revista de Investigación Educativa 24

enero-junio, 2017 | ISSN 1870-5308 | Xalapa, Veracruz

Instituto de Investigaciones en Educación | Universidad Veracruzana

Utilidad de la metodología científica en la investigación educativa del profesorado en formación: reflexión a partir de un estudio de caso en Ciencias Sociales sobre atención a la diversidad

Usefulness of scientific methodology in educational research in
teacher training: reflection from a case study in Social Sciences on
attention to diversity

Mtro. Ángel Ignacio Aguilar Cuesta

Doctorando

Universidad Autónoma de Madrid, España angelignacioaguilar@gmail.com

Dr. Daniel David Martínez Romera

Profesor

Universidad de Málaga, España

ddmartinez@uma.es

Desde el curso académico 2009/10 en España, es necesario poseer un Máster Oficial en Formación del Profesorado para ejercer la docencia en secundaria. Con ello se abre la posibilidad de trabajar contenidos sobre investigación educativa, tradicionalmente menos desarrollados que las aproximaciones didácticas innovadoras. Se pretende ilustrar dicha pertinencia mediante la revisión teórico-práctica del diseño e implementación del trabajo de campo de un profesor en formación, realizado como parte de su trabajo final de Máster en la especialidad de Ciencias Sociales, y cuyo objetivo era conocer la eficacia actual de la atención a la diversidad, fundamentalmente desde la óptica del rendimiento académico, en los centros escolares. De aquí se desprenden algunas claves relevantes sobre los beneficios que aporta una aproximación científica a la investigación educativa, como son la univocidad, la pertinencia y la factibilidad; así como se subraya la necesidad de fortalecer las destrezas de investigación en las nuevas generaciones docentes.

Palabras clave: Enseñanza universitaria; investigación educativa; formación de profesores; ciencias sociales; diversidad.

Recibido: 26 de enero de 2016 | Aceptado: 23 de noviembre de 2016 
Since 2009/10 course in Spain, it is necessary to hold a Master's degree in Teacher Training in order to practice high school education. This opens the possibility of adding content on educational research that transcend, traditionally less developed than innovative didactics. It is intended to illustrate that relevance through theoretical and practical review of the design and implementation of field work of a teacher in training, conducted as part of his final work of Master in the specialty of Social Sciences, whose objective was to understand the current effectiveness attention to diversity, mainly from the perspective of academic performance, in schools. From this analysis some important clues about the benefits of a scientific approach to educational research emerge, such as uniqueness, relevance and feasibility; and stresses the need to deepen the teaching of this type of content in the new generations.

Keywords: University education; educational research; teacher training; social sciences; diversity.

\title{
Utilidad de la metodología científica en la investigación educativa del profesorado en formación: reflexión a partir de un estudio de caso en Ciencias Sociales sobre atención a la diversidad
}

\author{
Usefulness of scientific methodology in educational \\ research in teacher training: reflection from a case \\ study in Social Sciences on attention to diversity
}

\section{Introducción: objetivo y objeto de investigación}

$\mathrm{E}_{\mathrm{n}}^{\mathrm{l}}$ objetivo fundamental del presente trabajo es subrayar la necesidad de una forprofesorado. Dicho planteamiento obtiene su fundamentacion en dos factores normativos clave:

La reformulación por parte del Estado del grado de exigencia científico-pedagógica sobre los nuevos docentes, mediante la conversión del tradicional curso de adap- 
tación pedagógica en un Máster Universitario Interdisciplinar, lo que permite abrir las puertas a nuevos grados de profundización en los diversos aspectos que definen la profesión docente. Y la existencia, dentro de la normativa docente de las Comunidades Autónomas, de figuras poco proyectadas como la de grupo de investigación no universitario (Junta de Andalucía, Decreto 55/2007 y Orden de 30/10/2007), y recibidas con marcadas reservas por los docentes noveles durante su proceso de formación.

En este sentido, e introduciendo una perspectiva práctica sobre el objetivo, el presente artículo ha tomado como base el instrumento de recogida de datos, creado al efecto dentro del trabajo final de Máster realizado por un alumno en la especialidad de Ciencias Sociales, y cuyo objeto de investigación principal se centra en el estudio de campo sobre la situación de la atención a la diversidad en varios centros educativos andaluces. Será sobre dicho instrumento, su formulación, su adecuación y su pertinencia, como se tratará de ilustrar la utilidad de una formación en investigación rigurosa. Ya que, entendemos, si no las precede ésta, tanto diagnóstico como intervención se vuelven fútiles.

Pocos aspectos son tan controvertidos y condicionantes en educación como los referentes a la definición y determinación de los subgrupos de estudiantes dentro de una comunidad educativa (Valls, Torre \& Siles, 2011). Si bien es cierto que las posturas normativas estatales y autonómicas parten de la asunción de la atención a la diversidad como un proceso de normalización inclusivo (tanto en Ley Orgánica de Educación [LOE] como Ley Orgánica para la mejora de la calidad educativa [LOMCE]), también contempla excepcionalmente la definición de subgrupos específicos, exclusivos, por motivos de adecuación a la singularidad previa diagnosis. En línea con el refinamiento de categorías establecido por LOMCE al respecto: necesidades educativas especiales, altas capacidades intelectuales, atención a la diversidad por ciclos educativos, programa de mejora del aprendizaje y del rendimiento, atención al alumnado de incorporación tardía y atención a la diversidad en los equipos docentes.

A esto se debe añadir que sólo recientemente (Benito \& González, 2013) se ha podido confirmar la influencia del efecto composición, por el que, al margen de las características individuales y familiares, la interacción mutua de los alumnos en los centros educativos y las aulas tiene una repercusión medible en el rendimiento académico individual.

Entendida como una convicción integradora de la heterogeneidad inherente a la sociedad contemporánea, la atención a la diversidad presenta, no obstante, una nada desdeñable diversidad de matizaciones en sus políticas públicas en el contexto espa- 
ñol (Abós \& Domingo, 2013). Desde énfasis claramente diferenciados entre colectivos (inmigrantes, altas capacidades, TDAH, entorno social...), a las estrategias de aula contempladas (agrupaciones flexibles, desdoblamientos, adaptaciones curriculares, apoyo...), o hasta la propia determinación de los factores de desigualdad, en los que llama la atención la escasa incidencia de los aspectos geográficos (residencia) y económicos (2013).

La tendencia normativa, más allá de la viva polémica que suscita en aspectos tan sustantivos como la posible segregación escolar o no (Andrés, 2013), dota de carácter aglutinante el concepto y ha hecho que la compensación educativa pase, de facto, a ser parte del mismo prisma inclusivo con la intención de buscar una mayor igualdad de resultados y expectativas (Araque \& Barrio, 2010). Esto, unido a la profusión legislativa que caracteriza al sistema educativo español, hace muy difícil para los centros actuar desde su propia autonomía, tanto más cuanto que las matizaciones de interpretación de algunos conceptos centrales, como el que nos ocupa, no son todo lo unívocos que debieran en su transición desde la promulgación estatal hasta su concreción autonómica: según la región encontramos instrumentos principales tan distintos como las guías de orientación, planes de absentismo, aulas virtuales, contratos-programa... Por tanto, es lógico que el grado y los criterios de control o seguimiento muestren diferentes niveles de materialización.

\section{Contexto científico y educativo}

Hablar de investigación científica en el marco de la educación española es siempre algo delicado desde una perspectiva histórica. Aceptando como punto de partida la creación del Instituto de Pedagogía San José de Calasanz en 1941, y la posterior red CENIDE-ICE (Centro Nacional de Investigación y Desarrollo Educativo-Institutos de Ciencias de la Educación), como efecto de la Ley General de Educación de 1970 (Jefatura del Estado, 1970), se puede afirmar que, siguiendo a De la Orden (2014), progresivamente la calidad y seriedad en la investigación educativa es notablemente superior en la actualidad, y se encuentra en pleno proceso de perfeccionamiento, aun cuando ésta tenga que hacerse en muchas ocasiones al margen de los propios Departamentos de los centros educativos.

Pero también es una cuestión delicada desde una perspectiva epistemológica, como señala Gurdián (2011), en tanto que deben converger factores que no siempre 
están presentes en este ámbito: la rigurosidad metodológica, basada en la buena formulación del problema y la forma de profundizar en él con objeto de realizar una diagnosis útil o una propuesta de solución o mejora; la exploración del universo de referencia, entendiendo por esto la obtención significativa de datos mediante una o varias técnicas y que supone una nada desdeñable inversión en tiempo y recursos; la naturaleza humana del objeto de estudio, aunque el comportamiento humano puede ser tabulado en patrones, este proceso no está exento de error ni contempla la posibilidad de transformación de la conducta durante el proceso de investigación, fruto de la interacción investigador-investigado.

De una forma más detallada y argumentada, estos puntos de referencia entroncan con el planteamiento de Méndez (2013), cuando afirma que hay, al menos, cinco grandes singularidades en la investigación educativa:

Exterioridad de los hechos sociales. Pese a lo tentador que puede resultar concebir el centro educativo, o el aula, como un pequeño laboratorio de experimentación aislado, lo que facilitaría en gran medida la investigación, nada más alejado de la realidad. Las personas que allí convergen traen consigo influencias exteriores que condicionan el desarrollo de las dinámicas internas en el centro o el aula.

Complejidad de los fenómenos humanos. A diferencia de lo que sucede con el comportamiento de la materia en el ámbito natural, la psicología humana es plástica en cuanto a su manera de percibir, procesar y posicionarse respecto a las influencias externas. Razón por la que la aplicación de un mismo método educativo, en clases o centros semejantes entre sí, no tiene por qué dar resultados similares, o interpretables bajo una misma óptica.

Influencia del cambio (tiempo) en los mismos. Durante el tiempo de existencia de una molécula, sus características, medidas en condiciones normales, son constantes; algo que para nada ocurre entre el alumnado, cuyo proceso de evolución cognitivo es, precisamente, muy dinámico desde la infancia hasta, al menos, el inicio de la edad adulta (Piaget, 1975, 1978).

Dificultad de establecer proyecciones (tendencias de comportamiento). En parte derivado de lo anterior, supone el reconocimiento explícito de que todo lo humano es contingente, evocando el famoso adagio de Vidal de La Blache. La evolución individual, y el comportamiento grupal derivado, se imbrican de forma compleja y sinérgica pero no determinista (Haken, 1984; Morin, 2006).

Transferencia, dialéctica, de valores en el proceso de investigación. La interacción entre observador y observado dista mucho de ser inocua en cualquier contexto; en el 
ámbito de la educación, procesos subconscientes de empatía, prejuicios o el contraste ideológico son, entre otros factores, causantes de la pérdida de imparcialidad tanto en la selección del objeto de estudio, el enfoque sobre el mismo, el proceso de recogida de datos, su análisis y la propuesta final de soluciones.

Muchas de las singularidades citadas son comunes a prácticamente cualquier tipo de investigación rigurosa. No obstante, el ámbito educativo hace especialmente sensible su tratamiento, ya que es difícil separar el ideal de sociedad del condicionamiento necesario para conseguirlo, a través de las estructuras educativas; esto aboca, irremediablemente, a considerar la dimensión política de individuos y colectivos, como una dificultad añadida a considerar en las investigaciones. Se subraya, así, la importancia de una adecuada formación epistemológica, técnica y procedimental, también para los conocimientos humanos y sociales.

Esto introduce la necesidad de comprender los cambios normativos recientes en el sistema educativo español, algo que, como reconoce Garrido (2005), no se puede hacer contentando a todo el mundo, por lo que nos centraremos en la exposición sucinta de hechos más que en la valoración de los mismos.

Tras un periodo de lenta evolución normativa, iniciado con el final de la dictadura del General Franco, en 1990 La Ley Orgánica de Ordenación General del Sistema Educativo (LOGSE) (Jefatura del Estado, 1990) se convierte en la primera ley que aborda, claramente, las cuestiones pedagógicas y didácticas. Tratándolas con un peso específico a la par, o incluso por encima, de los contenidos epistemológicos, y que aborda aspectos como las desigualdades, la integración o las necesidades especiales. En todo caso, supuso un cambio sustancial respecto a modelos pretéritos, no exento de críticas y detractores. La crítica más reconocida es el hecho de que se construyera contando sólo con la comunidad docente universitaria, y no la de otros niveles educativos.

Más allá de cuestiones centradas en la posesión de la verdad sobre el devenir educativo en dicho periodo, la instrumentalización de la educación como arma política había quedado establecida, siendo cada vez más evidente en las sucesivas leyes. Como ejemplo, la Ley Orgánica de Calidad de la Enseñanza (LOCE), de 2002, que no llegó a entrar en vigor y fue aglutinada por las venideras, volvió a repetir el modelo de ley creada sin consenso político amplio, como reacción a los informes nacionales e internacionales sobre la mala realidad formativa de los estudiantes españoles.

Se llega así a la situación actual, centrada en la Ley Orgánica de la Calidad de la Enseñanza (LOCE) (Jefatura del Estado, 2006), y su transición hacia la Ley Orgá- 
nica para la Mejora de la Calidad Educativa (LOMCE) (Jefatura del Estado, 2013), a expensas de lo que acabe sucediendo en los primeros compases del nuevo gobierno en minoría, ya que la aritmética parlamentaria determinará en los próximos meses si se ratifica la implantación, su grado de modificación o su anulación, según los escenarios conocidos posibles en el momento de la presente. En todo caso, esta situación se debe a que son leyes promulgadas por partidos gobernantes opuestos, sin consenso amplio, por lo que han sido muy criticadas y llevadas a derogaciones o modificaciones sustanciales a cada llegada de partido gobernante de signo distinto al anterior, y en las que las Competencias Básicas (ССвВ), conjunto de destrezas, conocimientos y actitudes útiles para la vida en sociedad, constituyen uno de los elementos menos discutidos en ambas propuestas (pasando, por agregación, de 8 en 2006 a 7 en 2013). Más allá de las discrepancias formales, ambos marcos normativos pretenden relanzar y mejorar lo que sí es un hecho consensuado, que la educación española tiene mucho margen para la mejora hasta equipararse con los modelos de éxito internacionales.

Informes internacionales como los de la Organización para la Cooperación y el Desarrollo Económico (ocDE 2015, 2016), la Organización de las Naciones Unidas para la Educación, la Ciencia y la Cultura (UNESCO, 2016), así como los estudios realizados por la International Association for the Evaluation of Educational Achievement (IEA, 2015, 2016), ponen de relieve el atraso relativo, y no tan relativo, de la formación final de la población española en relación con su contexto, siendo especialmente significativa la debilidad en cuanto a destrezas científicas y de comprensión lingüística. Hecho que subraya, bajo nuestro criterio, la pertinencia de investigar y mejorar en cuanto a formación epistemológica y técnica de los futuros docentes.

\section{Metodología}

El estudio responde a una metodología crítico-racional (Beltrán, 1985) aplicada a la encuesta de campo del trabajo base ya citado, cuyo objetivo era comprobar el grado de convergencia entre la normativa sobre atención a la diversidad y la situación práctica en los centros.

Presenta un carácter cualitativo y, en menor medida, cuantitativo, en tanto que ambas dimensiones confluyen en el instrumento original objeto de revisión. El proceso de estudio se articula alrededor de tres aspectos secuenciales: establecimiento y fundamentación de criterios objetivos de análisis, aplicación de los mismos a la pro- 
puesta inicial y reformulación del instrumento para satisfacer el carácter científico del mismo.

Los tres criterios a seguir serán los definidos por Quivy y Van Campenhoudt (2005), en relación a las características deseables en una buena pregunta de investigación:

En primer lugar, ha de tener una única forma de ser interpretada (univocidad, o claridad). Esto hace posible obtener la información que se pretende recabar, y es un primer filtro para evitar preguntas vacuas, ilusorias o retóricas.

En segundo lugar, ha de ser factible, esto es, debe existir una concomitancia entre la naturaleza de la información, el universo de referencia y la capacidad de la investigación para construir los datos. Ya que realizar una buena pregunta que no puede ser contestada o una hipótesis que no puede ser comprobada carece de utilidad científica.

En tercer lugar, ha de ser pertinente, esto es, evitará condicionar u orientar al entrevistado en sus respuestas. Este aspecto suele ser objeto de errores recurrentes en las investigaciones en Humanidades y Sociales, ya que el grado de condicionamiento mutuo en el binomio observador-observado no es sólo de naturaleza física, como ocurre en la investigación experimental, sino también psicológica. Usos apologéticos, o de diatriba, son muy habituales en el empleo de la investigación con entrevistas y encuestas.

Sobre la muestra, los datos recogidos en el sondeo pertenecen a ocho centros educativos: un centro rural, otro periurbano y cinco urbanos, pertenecientes a tres provincias $^{1}$ distintas (Granada, Málaga y Sevilla), con una población encuestada en la que $70 \%$ ejercía funciones docentes y $30 \%$ directivas o mixtas; esto garantizó que tanto la óptica práctica como institucional quedara reflejada.

Además, aunque claramente desequilibrada, la existencia de encuestados de centros públicos $(80 \%)$ y $\operatorname{concertados}^{2}(20 \%)$ abría la puerta al análisis comparado; no obstante, la heterogeneidad en cuanto a su oferta educativa (4 categorías, desde sólo Educación Secundaria Obligatoria (ESO) hasta incluir todos los niveles preuniversitarios) invita a la reflexión sobre cómo simplificar un análisis cuyo rango de edad estudiantil es tan amplio, y cuyos condicionantes psicopedagógicos son tan cambiantes (especialmente desde primaria a bachillerato y formación profesional).

1. Semejantes, en cuanto a escala de organización territorial, al municipio mexicano.

2. Centros de titularidad privada pero subvencionados por el erario público, con objeto de que realicen una gestión pública de las peticiones de matriculación estudiantil. 


\section{Utilidad de la metodología científica en la investigación}

educativa del profesorado en formación

Ángel Ignacio Aguilar Cuesta, Daniel David Martínez Romera

\section{Análisis del instrumento inicial}

El primer cuestionario se compone de un total de diecisiete preguntas, organizadas en varios bloques lógicos no secuenciales: identificación del centro y funciones del docente entrevistado, descripción de magnitudes (población estudiantil, ratios, oferta educativa), políticas de actuación del centro para las agrupaciones de clase, valoración de las actitudes discentes en clase, de los recursos disponibles para gestionar la atención a la diversidad, así como cuestiones para el juicio de valor sobre la situación educativa. El instrumento así organizado quedó como se muestra en la Tabla 1.

\section{Tabla 1. Estructura original del cuestionario}

\begin{tabular}{|c|c|}
\hline 1 & Centro en el que se trabaja. \\
\hline 2 & Ratio de alumnos/as. \\
\hline 3 & $\begin{array}{l}\text { Número de alumnado que accede desde la Educación Primaria Obligatoria (EPO) sin los } \\
\text { contenidos ni los objetivos mínimos marcados por la legislación vigente. }\end{array}$ \\
\hline 4 & Número de alumnado con perfil activo. \\
\hline 5 & Número de alumnado con perfil pasivo (no afecta a la dinámica de clase). \\
\hline 6 & Número de alumnado con perfil disruptivo (afecta a la dinámica de clase). \\
\hline 7 & $\begin{array}{l}\text { Número de alumnado que consigue finalizar la ESO habiendo desarrollado todos los } \\
\text { contenidos y objetivos mínimos exigidos. }\end{array}$ \\
\hline 8 & $\begin{array}{l}\text { Porcentaje de alumnado con desfase curricular que logra adquirir todas las competencias y } \\
\text { contenidos mínimos al terminar la ESO. }\end{array}$ \\
\hline 9 & Tipo de agrupamiento dentro del aula. \\
\hline 10 & Tipo de agrupamiento con el alumnado que no promociona de curso. \\
\hline 11 & Realización de agrupamientos flexibles, medidas de apoyo en clase o grupos específicos. \\
\hline 12 & Número de recursos disponibles en el centro. \\
\hline 13 & ¿Se ha conseguido trabajar e integrar las competencias de forma integral? \\
\hline 14 & Apartados que se utilizan para segregar. \\
\hline 15 & Creación de centros para la élite intelectual. \\
\hline 16 & Homogeneización de los conocimientos. \\
\hline 17 & Medidas políticas para paliar la segregación. \\
\hline
\end{tabular}


Para comprender la intencionalidad de esta propuesta es necesario citar las motivaciones del docente en formación: parte de la asunción vivida durante la fase de docencia en prácticas, durante la cual vislumbró la existencia de un comportamiento segregador, al observar cómo estaba organizado el alumnado según criterios académicos dentro de las aulas. Entendió necesario conocer las causas de la consecuencia que observó y explica su primera orientación en bloques del cuestionario: el papel de los docentes, la concepción de la atención a la diversidad en los mismos, su valoración sobre la normativa al respecto y el papel de las competencias básicas como garantía de mínimos en contextos escolares no homogéneos.

Con el instrumento de recogida definido en estos términos, los datos de encuesta reflejaron como quejas más frecuentes la falta de medidas políticas efectivas para detener la segregación en las aulas y el déficit de recursos disponibles; también que, siempre según el valor medio de las respuestas ofrecidas por docentes y directivos, $20 \%$ del alumnado que llega a los centros de secundaria lo hace sin las competencias y los contenidos mínimos necesarios de la etapa anterior. Por ello, se concluyó en un primer análisis, parecía que las medidas para corregir los desfases curriculares no estaban siendo eficaces, debido a la falta de inversión, de profesorado y de recursos, como factores clave más reiterados.

Los resultados de la encuesta, en todo caso, no confirmaban de manera irrefutable la existencia, o no, de procesos de segregación entroncados con una gestión parcial de la atención a la diversidad, por lo que la adecuación entre la situación en los centros y la normativa vigente que la encuesta pretendía ayudar a determinar, no pudo quedar definida. Varias razones se abren aquí como argumentos explicativos:

La insuficiencia de la muestra, ya que ni el número de centros ni de personas encuestadas permite hacer una extrapolación autonómica, al no ser representativa. El problema de base aquí es la formulación de una pregunta no factible, pues se carecía de los recursos necesarios para comprobarla, por parte del docente en formación. Ateniéndonos a las dimensiones del universo de referencia educativo en Andalucía para el curso 2014/15 una muestra del $2 \%$, por ejemplo, habría implicado más de 130 centros y 1,200 docentes. Números que se alejan en órdenes de magnitud con los datos recolectados.

Errores de diseño en la encuesta utilizada, incluyéndose de manera secundaria aspectos como los tipos de tabulación y las escalas de medición asociadas. Se trata de un aspecto en el que sí se puede profundizar, y cuyo proceso de mejora permite ilustrar, desde un punto de vista didáctico, cómo debe orientarse una investigación educativa rigurosa. Y constituye el tercer paso. 
Se detectó la ausencia de algunas cuestiones clave relacionadas con la implicación de la persona encuestada en el centro y la tipología del mismo (preguntas 2 y 5), cruciales para explorar la existencia de patrones de comportamiento ligados a la función de quien responde o del propio centro. Lagunas que cuestionaban la claridad de dichas preguntas.

Además, contar con especialistas como fuente de información y no definir mecanismos para recabar su posición respecto a la situación educativa general, así como la del propio centro, se consideró también una carencia a subsanar (preguntas 19 y 2o). Con ello se centra el juicio de valor en el entrevistado, evitando problemas de pertinencia.

Algunas de las cuestiones del formulario original (preguntas 7, 14 a 17) fueron suprimidas o reformuladas e incorporadas en preguntas equivalentes del nuevo cuestionario. Bien fuese para orientar de forma más clara el instrumento al objetivo de la investigación (7), bien para encontrar un mayor rigor científico en la formulación de las preguntas en términos de claridad, factibilidad y pertinencia.

Finalmente, se realizó un tercer grupo de modificaciones encaminadas a obtener información sensible de la forma menos agresiva posible, desdoble de la pregunta original 6, pero introduciendo una nueva secuencia de preguntas, de cuya respuesta se puede deducir algo no preguntado explícitamente: el porcentaje de alumnado disruptivo en el aula. Y una revisión de los valores nominales posibles en las preguntas tabuladas, así como una estandarización de escala de intensidad (1 a 10) para las preguntas de dicho tipo. Con todo ello se pretende hacer más concreta y menos confusa la respuesta, fortaleciéndose en última instancia comprobar la intención de partida que motivó el cuestionario.

\section{Reformulación y resultados}

El cuestionario obtenido tras el análisis (Tabla 2), exhibe de forma más nítida las dos características que este tipo de refinamiento persigue: obtener información útil para el tema de estudio y hacerlo evitando en la población encuestada situaciones que puedan socavar la calidad y veracidad de las respuestas. 


\section{Tabla 2. Estructura revisada y equivalencia original (segunda columna)}

\begin{tabular}{|c|c|c|}
\hline 1 & 1 & Indique el nombre y dirección del centro en el que trabaja \\
\hline 2 & - & En el marco de la educación reglada, su función principal es... \\
\hline 3 & 1 & ¿A qué tipo de centro se encuentra vinculado? \\
\hline 4 & 1 & ¿Cuál es su oferta educativa? \\
\hline 5 & - & En cuanto a la oferta educativa, su centro... (compensatoria, bilingüismo, especiales...) \\
\hline 6 & 6 & $\begin{array}{l}\text { Indique cuál es, a su criterio, el grado de conflictividad interna del alumnado en su } \\
\text { centro educativo... }\end{array}$ \\
\hline 7 & 6 & $\begin{array}{l}\text { Indique cuál es, a su criterio, el grado de conflictividad externa del alumnado en su } \\
\text { centro educativo... }\end{array}$ \\
\hline 8 & 2 & ¿Cuál es la ratio alumnos/aula en su centro? \\
\hline 9 & 1 & ¿En qué área de conocimiento imparte su docencia? \\
\hline 10 & 4 & $\begin{array}{l}\text { Considerando su experiencia reciente, ¿qué porcentaje del alumnado estima que asiste } \\
\text { a clase con regularidad y actitud positiva hacia el aprendizaje? }\end{array}$ \\
\hline 11 & 5 & Del alumnado restante, ¿qué porcentaje presenta un perfil pasivo y no disruptivo? \\
\hline 12 & 3 & En su centro, ¿cómo de significativa es la población estudiantil con desfase curricular? \\
\hline 13 & 8 & $\begin{array}{l}\text { ¿En qué proporción se consigue reintegrar y normalizar su desarrollo académico, } \\
\text { alcanzando los objetivos mínimos exigidos? }\end{array}$ \\
\hline 14 & 9 & $\begin{array}{l}\text { En su centro, ¿qué criterio se aplica para realizar el reparto general de alumnos por } \\
\text { aula? }\end{array}$ \\
\hline 15 & 10 & Y en relación al alumnado repetidor, ¿cómo se realiza su distribución? \\
\hline 16 & 11 & $\begin{array}{l}\text { ¿Se realizan en su centro agrupamientos flexibles, grupos específicos o medidas de } \\
\text { apoyo en clase? }\end{array}$ \\
\hline 17 & 12 & $\begin{array}{l}\text { ¿Cuenta con los recursos y herramientas necesarios para el desarrollo de su docencia en } \\
\text { el aula? }\end{array}$ \\
\hline 18 & 13 & $\begin{array}{l}\text { Atendiendo a su experiencia, ¿en qué medida se trabaja en las aulas siguiendo el Plan } \\
\text { de Centro por encima de las exigencias estrictamente de contenidos de cada materia? }\end{array}$ \\
\hline 19 & - & $\begin{array}{l}\text { Considerando su trayectoria docente y discente, ¿cuál ha sido la evolución de la } \\
\text { educación en las aulas? }\end{array}$ \\
\hline 20 & - & $\begin{array}{l}\text { Si tuviera la ocasión de hacer valer sus ideas y experiencia, ¿qué actuaciones llevaría a } \\
\text { cabo para mejorar la eficiencia de los centros educativos? }\end{array}$ \\
\hline
\end{tabular}

Uno de los aspectos más relevantes del nuevo cuestionario ha sido su habilidad para centrar el universo de referencia en contextos especialmente significativos de la atención a la diversidad; así, 70\% de los encuestados pertenecía a un centro acogido a un 
Plan de Compensación Educativa, ${ }^{3} 10 \%$ a centros bilingües ${ }^{4}$ y $20 \%$ no tenía singularidad específica al respecto (Figura 1).

¿Cuál es su oferta educativa?

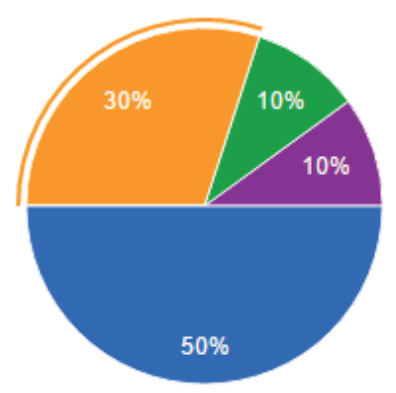

Secundaria

Primaria + Secundaria

Secundaria + FP

Todos los niveles preuniversitarios

Otro

En cuanto a la oferta educativa, su centro...

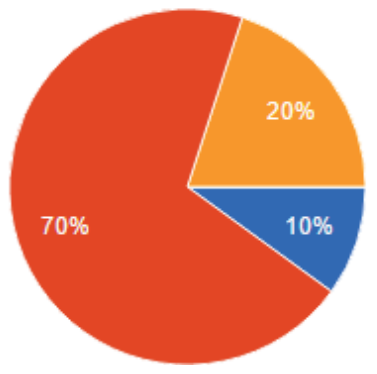

Es bilingüe

Está acogido a un Plan de

Compensación Educativa

No está acogido a plan o proyecto

específico

Otro

\section{Figura 1. Oferta educativa y planes específicos}

Fuente: Elaboración propia.

El primer hecho relevante que permite corroborar la encuesta es el comportamiento diferencial de la conflictividad interna del alumnado según el grado de desarrollo ur-

3. Centros que, por las características socioeconómicas desfavorables de su población estudiantil, reciben un tratamiento específico por las administraciones públicas.

4. Con enseñanza en Español e Inglés en todas las materias del currículo. 
bano de su contexto, siendo los centros urbanos y periurbanos los que arrojan valores más altos, y nulos o poco significativos en el resto.

Al abordar el grado de conflictividad externa del alumnado, hacia docentes y personal del centro, se observó un comportamiento análogo, si bien es posible detectar un mayor grado de dispersión por parte de los docentes dentro de los mismos centros, y también en relación a la perspectiva centro-periferia expuesta anteriormente.

Evidentemente, factores no controlados por la encuesta, como la personalidad y el estilo docente de cada encuestado, así como el grado de integración comunitaria de cada centro, deberían investigarse antes de aventurar interpretaciones en firme. Un factor muy relevante, en cambio, sí fue registrado como elemento contextualizador de la realidad de cada entrevistado, la ratio de alumnos por aula, lo que refleja una situación claramente heterogénea, con valores que van de los 12 a los 30 alumnos, y en el que la inmensa mayoría se mueve por encima de los 24 alumnos (80\%). La capacidad del docente para gestionar su clase y desplegar recursos y propuestas didácticas de atención a la diversidad es claramente distinta entre ambos extremos.

En las preguntas relacionadas con la evaluación docente del alumnado, la dispersión de los valores fue igualmente importante. Así, la pregunta 10 arroja valores extremos de $15 \%$ y $98 \%$, con $40 \%$ de valoraciones por debajo del umbral del $50 \%$, lo que permite hacerse una idea de las percepciones tan distintas al respecto. Para poder explicar dichas situaciones polares entendemos que sería necesario desagregar los datos a nivel de curso académico, en lugar de centrarlos en la valoración global de la docencia impartida por el docente, algo que trasciende las premisas del estudio y plantea dificultades de confidencialidad estadística.

En cuanto a alumnado no motivado pero no conflictivo restante (pregunta 11), la situación estrecha algo su espectro, con límites que oscilan entre 10\% y 65\%; para aprovechar el sentido de esta cuestión es necesario considerar la siguiente, 12, en tanto que al obtener un porcentaje de alumnos con población estudiantil con desfase curricular se puede realizar una aproximación grosera al volumen de alumnos con necesidades específicas de atención, siguiendo la lógica: alumnado de clase menos alumnado motivado y participativo es igual a alumnado desconectado.

Y a su vez: alumnado desconectado - alumnado con desfase curricular = alumnado invisible, es decir, alumnado que no se integra al ritmo de la clase, pero que por su comportamiento no conflictivo y su capacidad para seguir el ritmo no es objeto de atención especial. Gracias al silogismo de preguntas recogido en el cuestionario, se hace posible considerar esta nueva forma de interpretar la realidad del aula con objeto 
de mejorar las acciones didácticas y de integración en la misma. Esto es especialmente importante en tanto que el grado de reintegración del alumnado con desfase curricular no alcanza 50\% de este alumnado según 70\% de los encuestados.

Las causas de esta situación guardan una clara relación, entre otros aspectos a considerar, con la forma de configurar las aulas: $50 \%$ de los entrevistados sostiene que el criterio para ocupar las clases de los diferentes cursos es el rendimiento académico, seguido de un extrañamente alto porcentaje de docentes que prefirieron no especificarlo o fueron demasiado laxos en su concreción para poder categorizar su respuesta (40\%), y en último lugar, $10 \%$ basado en la personalidad del alumnado. La naturaleza de los datos obliga a reflexionar sobre esta pregunta, ya que parece claro que aquí se ha dado con un elemento sensible que exige probablemente un mayor desglose y circunloquio metodológico.

Relativamente más sencillo es, por el contrario, obtener información sobre el alumnado repetidor, que es asignado con base en tres criterios fundamentales: $30 \%$ afirma que se realiza por distribución equitativa entre clases, otro $30 \%$ por concentración en un aula y $10 \%$ de forma aleatoria; esto deja un cajón de sastre de 30 puntos porcentuales que obliga a reflexionar en los términos de la pregunta anterior. De forma casi antagónica, la realización de grupos flexibles es una práctica habitual o puntual para $90 \%$ de los entrevistados, con una distribución de $70 \%$ y $20 \%$, respectivamente (Figura 2).

Esto lleva a considerar, entre otras variables, si la forma de asignación inicial no podría ser más coherente con la realidad de los centros educativos: como hemos señalado, estos muestran una diversidad latente tanto para los alumnos con adaptaciones curriculares como sin ellas; sin embargo, la realidad de los grupos flexibles, a tenor de los resultados de reintegración, parecen no ser suficientes. De esta forma, modificar la agrupación del alumnado se erige como un elemento de efectividad cuestionable, salvo por la postura socialmente institucionalizada de que la "bajada del rendimiento" encuentra su causa en la influencia perniciosa que tienen los alumnos no modélicos entre aquellos con mejores calificaciones o valoración académica, realizando así una interpretación sesgada del efecto composición que abordamos en el primer epígrafe. 
Utilidad de la metodología científica en la investigación educativa del profesorado en formación

Ángel Ignacio Aguilar Cuesta, Daniel David Martínez Romera

En su centro, ¿qué criterio se aplica para realizar el reparto general de alumnos por aula?

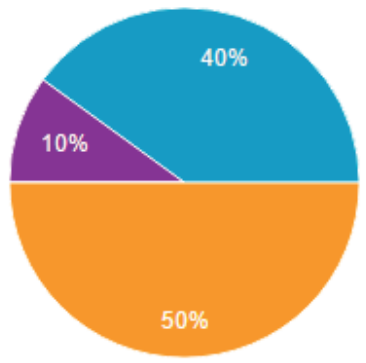

Ninguno, puramente aleatorio Centro o lugar de procedencia

Rendimiento académico previo

Implicación de las familias

Personalidad del alumnado

Otro

Y en relación al alumnado repetidor, ¿cómo se realiza su distribución?

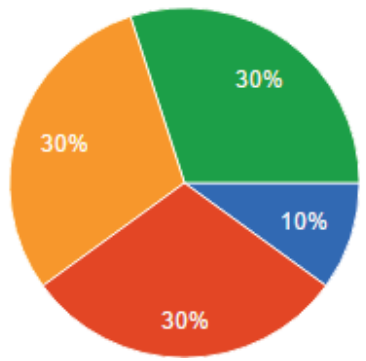

De forma aleatoria

Reparto equitativo entre todas las clases

Concentración en una clase

Otro

Figura 2. Atención a la diversidad, criterios de agregación

Fuente: Elaboración propia.

\section{Discusión}

Los resultados obtenidos en la segunda encuesta, además de ofrecer una mayor claridad de interpretación, abren la posibilidad a nuevos enfoques analíticos como el de centro-periferia, en relación a la posibilidad de analizar diferenciadamente el comportamiento de centros rurales, periurbanos y urbanos, al ofrecer un mayor nivel de desagregación analítica. En todo caso, los resultados supusieron una clara mejora en 
cuanto a la calidad de los mismos en relación a la encuesta pretérita, como trataremos de ilustrar seguidamente.

Obviamente, este ejercicio de reflexión analítica y metodológica deja entrever que parte del alumnado de nuestras aulas se dispersa de forma temprana, sin posibilidad de reintegración en un alto porcentaje. Por ende, cabe preguntarse dónde está el límite en la intencionalidad y definición de grupos, una vez entendido que sus miembros poseen diferentes desarrollos y potenciales intelectuales (Gardner, 2011), entornos socio-familiares muy diversos (Bellei, 2013; Jaume, 2013), expresiones de la propia identidad que condicionan la integración en las dinámicas internas de la clase, y conviven con políticas educativas no siempre efectivas (Merino, 2013) o factores extraoficiales escasamente explorados (El-Habib, Jiménez, Ruiz \& Jareño 2016), entre otros aspectos.

La reflexión y mejora del instrumento, inspirada en la metodología CUADIVER (Cuestionario de Opinión sobre la Atención a la Diversidad) (Alonso, Castedo \& Juste, 2013; Varela \& Alonso, 2015) y la perspectiva orientadora de Miranda, Burguera y Arias (2015), han permitido dar un giro a la profundidad analítica del trabajo de campo, al facilitar la detección de nuevos aspectos de reflexión: según los datos recogidos de la respuesta a la pregunta 17 , los encuestados coinciden en la necesidad de una mayor dotación de recursos de apoyo a la docencia, aunque además, constatan en la cuestión 20 algunas líneas maestras compartidas por la mayoría: hay que reconectar con el alumnado pasivo, ya que son una de las principales dificultades para la marcha del ritmo de clase. Para ello, muchos encuestados optan por la dinamización de las clases con modelos distintos al de un profesor por clase, siendo recurrente la figura del profesor de apoyo; implicar a las familias como parte de la visión global de la enseñanza, y desarrollar dinámicas grupales más efectivas que permitan establecer vínculos positivos de aprendizaje.

En el lado más polémico, es posible encontrar posturas sobre la necesidad de establecer clases más homogéneas con base en el rendimiento académico, así como enfatizar una especialización curricular que trascienda la formación humanista reminiscente en el currículo. Bajo nuestro punto de vista, este segundo grupo de posturas supone una rémora educativa, aún más flagrante en centros acogidos a Planes de Compensación Educativa, en los que los docentes entrevistados afectados confirman tanto un abandono administrativo como el incumplimiento de la propia legislación asociada, al no satisfacer rigurosamente las dotaciones específicas desglosadas en el marco normativo vigente. 


\section{Conclusiones y propuestas de futuro}

Lo que comenzó como un pequeño estudio de campo, en el contexto de un Trabajo Final de Máster, sobre el problema de la atención a la diversidad dentro de las aulas de los centros malacitanos de educación secundaria, ha servido para ilustrar dos elementos críticos que es necesario trabajar en relación con las nuevas generaciones docentes, ya que se han mostrado claramente insuficientes.

De un lado, la necesidad de fortalecer el dominio epistemológico (Cardona, 2013; Herrero, 2012) de la materia a la que se enfrenta el profesorado novel, en este caso, la investigación educativa, con objeto de ayudar a articular su pensamiento de forma más efectiva de cara a posibles acciones que puedan desarrollar. Y de otro, fortalecer sus destrezas técnicas, entendidas como expresión instrumental de la primera, con objeto de hacer viable el voluntarismo de tantos docentes que se animan a querer conocer su contexto educativo para mejorarlo.

En efecto, el estudio de caso utilizado ha permitido ilustrar que no sólo una motivación positiva y comprometida es suficiente para mejorar la realidad educativa, como se percibía claramente en el cuestionario original, sino que además es necesario pensar bien cómo hacerlo (revisión), siguiendo unas pautas mínimas de rigor científico. Además, las diferencias encontradas entre ambas propuestas subrayan la importancia de la reflexión científica de una forma epistemológicamente integrada (Bachelard, 1976), en lo que nos concierne, en el ámbito de la investigación educativa.

Somos conscientes de la magnitud de la tarea que se abre ante nosotros, sin embargo, entendemos que este primer paso (señalar e ilustrar su importancia), permitirá sumar esfuerzos para la mejor formación en investigación educativa de nuestros docentes, lo que en última instancia permitirá que la sociedad se beneficie de unas acciones docentes y unos centros educativos más eficientes.

\section{Lista de referencias}

Abós, P., \& Domingo, V. (2013). La diversidad territorial de España ante el reto de la atención a la diversidad: diversidad de políticas públicas. Revista Electrónica Interuniversitaria de Formación del Profesorado, 16(2). doi:http://dx.doi. org/10.6018/reifop.16.2.182751 
Alonso, J. D., Castedo, A. L., \& Juste, M. P. (2013). Diseño y Propiedades Psicométricas de un Cuestionario de Opinión sobre la Atención a la Diversidad (CUADIVER). Psicologia: Reflexão e Crítica, 26(2), 270-286.

Andrés, F. (noviembre, 2013). La segregación escolar en nuestro sistema educativo. Forum Aragón: Revista Digital de FEAE-Aragón sobre organización y gestión educativa, 10, 47-52.

Araque, N., \& Barrio, J. L. (junio, 2010). Atención a la diversidad y desarrollo de procesos educativos inclusivos. Prisma Social: Revista de Ciencias Sociales, 4, 1-37.

Bachelard, G. (1976). El materialismo racional. Buenos Aires: Paidós.

Bellei, C. (2013). El estudio de la segregación socioeconómica y académica de la educación chilena. Estudios pedagógicos, 39(1), 325-345.

Beltrán, M. (1985). Cinco vías de acceso a la realidad social. Reis. Revista Española de Investigaciones Sociológicas, 29, 7-41.

Benito, R., \& González, I. (2013). ¿Puede el efecto composición reducir las desigualdades educativas? Una mirada sobre los efectos de la segregación escolar en España y los países del modelo de integración uniforme. Revista de la Asociación de Sociología de la Educación (RASE), 6(1), 49-71.

Cardona, J. (2013). Epistemología del saber docente. Madrid: Universidad Nacional de Educación a Distancia.

El-Habib, B., Jiménez, M., Ruiz, R., \& Jareño, D. (2016). Composición escolar y expectativas del alumnado por origen y etnia: una aproximación a la segregación escolar extraoficial. RASE: Revista de la Asociación de Sociología de la Educación, 9(1), 59-77. doi:10.7203/RASE.9.1.8404

Gardner, H. (2011). Frames of mind: The theory of multiple intelligences. Nueva York: Basic Books.

Garrido, M. (agosto, 2005). Historia de la educación en España (1857-1975). Una visión hasta lo local. Revista Contraluz, 2, 89-146.

Gurdián, A. (julio-diciembre, 2011). ¿Quién es el sujeto en la investigación educativa? Revista Electrónica Educare, 15(2), 7-21. Recuperado de http://www.revistas. una.ac.cr/index.php/edUCARE/article/view/1955

Haken, H. (1984). Sinergética: la doctrina de la acción de conjunto. Barcelona: Argos Vergara.

Herrero, C. (2012). Hacia una didáctica integrada de las Ciencias Sociales. Revista de Didácticas Específicas, 6, 140-158.

International Association for the Evaluation of Educational Achievement. (2015). 
Trends in International Mathematics and Science Study. Recuperado de: http:// www.iea.nl/our-studies

International Association for the Evaluation of Educational Achievement. (2016). Progress in International Reading Literacy Study. Recuperado de http://www. iea.nl/our-studies

Jaume, D. (2013). Un estudio sobre el incremento de la segregación escolar en Argentina (Documento de Trabajo Número 143). Argentina: Centro de Estudios Distributivos Laborales y Sociales.

Jefatura del Estado. (1970). Ley 14/1970, de 4 de agosto, General de Educación y Financiamiento de la Reforma Educativa. Boletín Oficial del Estado, 187, 1252512546.

Jefatura del Estado. (1990). Ley Orgánica 1/1990, de 3 de octubre de 1990, de Ordenación General del Sistema Educativo. Boletín Oficial del Estado, 238, 2892728942.

Jefatura del Estado. (2006). Ley Orgánica 2/2006, de 3 de mayo, de Educación. Boletín Oficial del Estado, 106, 17158-17207.

Jefatura del Estado. (2013). Ley Orgánica 8/2013, de 9 de diciembre, para la mejora de la calidad educativa. Boletín Oficial del Estado, 295, 97858-97921.

Méndez, D. (enero-febrero, 2013). La metodología científica y la investigación educativa. Revista Acta Universitaria, 23(1), 23-30.

Merino, R. (2013). Las sucesivas reformas de la formación profesional en España o la paradoja entre integración y segregación escolar. Education Policy Analysis Archives, 21(66). doi:http://dx.doi.org/10.14507/epaa.v21n66.2013

Miranda, M., Burguera, J. L., \& Arias, J. M. (2015). La atención a la diversidad en los centros de secundaria: diseño y propiedades de un cuestionario de opinión dirigido a orientadores. En Asociación Interuniversitaria de Investigación Pedagógica (Ed.), Investigar con y para la sociedad (pp. 103-112). España: Editor.

Morin, E. (2006). El Método IV: las ideas de las ideas. Madrid: Cátedra.

Orden de la, A. (2014). La investigación educativa en España. Antecedentes y perspectiva. Revista Participación Educativa, 3(5), 33-41. Recuperado de http://ntic. educacion.es/cee/revista/pdfs/nsart arturo orden.pdf

Organización de las Naciones Unidas para la Educación, la Ciencia y la Cultura. (2016). Global Education Monitoring Report 2016. Recuperado de http:// es.unesco.org/gem-report/

Organización para la Cooperación y el Desarrollo Económico. (2015). PISA in Focus. 
Recuperado de http://www.mecd.gob.es/inee/PISA-in-focus.html Organización para la Cooperación y el Desarrollo Económico. (2016). Panorama de la Educación. Indicadores de la OCDE 2016. Recuperado de http://www.mecd.gob. es/inee/publicaciones/indicadores-educativos/Indicadores-Internacionales/ OCDE.html

Piaget, J. (1975). Introducción a la Epistemología Genética. Buenos Aires: Paidós.

Piaget, J. (1978). La equilibración de las estructuras cognitivas: problema central del desarrollo. Madrid: Siglo XXI.

Quivy, R., \& Van Campenhoudt, L. (2005). Manual de Investigación en Ciencias Sociales. México: Limusa.

Valls, R., Torrego, L., \& Siles, G. (2011). Agrupación del alumnado y atención a la diversidad: entre la exclusión y la inclusión educativa. Papeles Salmantinos de Educación, 15, 181-197.

Varela, E. V., \& Alonso, J. D. (2015). Medidas de atención a la diversidad: incidencia y concepciones desde la inspección educativa. Revista de Estudios e Investigación en Psicología y Educación, 11, 5-8. doi:10.17979/reipe.2015.0.11.88 